\title{
Analysis of QoS for WiMAX and 3G networks with same and different speed using Qualnet 6.1
}

\author{
OsamahThamer Hassan AL-Zubaidi ${ }^{1}$, Rajeev Paulus ${ }^{2}$, A.K.Jaiswal ${ }^{3}$, \\ A.Ashok ${ }^{4}$, Ashish Shukla ${ }^{5}$ \\ $\left({ }^{1}\right.$ M.Techstudent, Dept. Of Ece, Shiats/Allahabad $),\left({ }^{2}\right.$ Assistant Prof Dept Of Ece,Shiats/Allahabad $),\left({ }^{3}\right.$ Prof. And \\ H.O.D Dept. Of Ece,Shiats/Allahabad),,,${ }^{4}$ Assistant Prof Dept Of \\ Ece,Shiats/Allahabad), $\left({ }^{5}\right.$ M.Techstudent,Dept.Of Ece,Shiats/Allahabad)
}

\begin{abstract}
New and increasingly advanced data services are driving up wireless traffic, which is being further boosted by growth in voice applications in advanced market segments as the migration from fixed to mobile voice continues. Meanwhile, difficult market conditions have caused a number of operators to delay making substantial investments in upgrading their networks to higher capacity technology. While many service providers and operators may be somewhat familiar with the previously mentioned $2.5 G$ services, they are now hearing about newer $3 G$ technologies such as UMTS and HSDPA, and other technologies such as WiMAX (IEEE 802.16e), which offer substantial improvements in data rate and spectral efficiency. This paper focuses on the technical differences between these technologies by comparing the differences between the two advanced technologies of wireless network: WiMAX and $3 G$ network based on mobility and without mobility. Thus several parameters like energy efficiency andQoS to evaluate their performance to find out which networks present the best services and advantages. The metrics used for performance evaluation are Average Jitter, Average Delay, Energy Consumption, Average Queue length, Signal Received with Errors, Total unicast received, Unicast received Throughput of the WiMAX and $3 G$ network using QualNet 6.1 simulator.
\end{abstract}

Keywords : WiMAX, 3G, QoS, CBR, QualNet.

\section{Introduction}

Wireless Networks has huge diversity ranging from Wi-Fi (802.11) covering small areas to WiMAX (802.16) that covers several miles. Wi-Fi is the industry name for wireless LAN (WLAN) communication technology related to the IEEE 802.11 family of wireless networking standards. Although the broadband data market segment has been rather anemic for the past couple decades, declining average revenue per user has caused carriers to look at wireless broadband data as a means to drive revenue growth. While many service providers and operators may be somewhat familiar with the previously mentioned $2.5 \mathrm{G}$ services, WiMAX (IEEE 802.16e), which offer substantial improvements in data rate and spectral efficiency. Service operators face some tough decisions as they witness a rising demand for "anywhere, anytime" broadband access. Serviceoperators sense that there is a real business opportunity to provide a substantial increase to the all-important average revenue per user (ARPU). This paper examines the different technological options facing a service provider considering deploying a personal, mobile, broadband access network. The paper provides a high level comparison of commonalities and differences of these various technologies. It focuses on high capacity technologies comparing WiMAX 4G technology and the different 3G options. Mobile Broadband Wireless Access (BWA) is introduced to provide flexible and cost effective solution. It has many advantages as high speed, flexibility and easier to scale. It has the potential to serve customers that are unsatisfied or unserved by wired broadband services. Interworking of the wireless networks requires different mobility management, security and QoS mechanisms to be harmonized and integrated into a common architecture. The main hindrance to its competitive edge is the lack of an infrastructure for the WiMAX Core Network (CN), which makes it impossible to become a head-to-head competitor against current cellular networking infrastructures.In the past, there has been a lot of research focusing on performance of the WiMAX and $3 \mathrm{G}$ performance. A Simulation and performance analysis of Ad-hoc routing protocol in Wi-Fi \&WiMAX networks using QualNet by declining average revenue per user has caused carriers to look at wireless broadband data as a means to drive revenue growth [1]. While many service providers and operators may be somewhat familiar with the previously mentioned. An understanding WiMAX and 3G for portable/Mobile Broadband Wireless by offer substantial improvements in data rate and spectral efficiency [2]. Mobile WiMAX as aNext Generation Broadband Wireless Networks by different technological options facing a service provider considering deploying a personal, mobile, broadband access network [4]. Loose coupling Approach for UMTS/WiMAX Integration by adaptive modulation technique in non-line-of-sight (NLOS) environments. Base stations of WiMAX can provide communication without the need of line-of-sight (LOS) connection [5]. Interworked WiMAX-3G Cellular Data Networks architecture for Mobility Management and Performance Evaluation by interworking environment and the service exibiity that this technology offers to the 
currently deployed wireless broadband technologies makes it appealing to users, service developers and network operators [6]. WiMAX-UMTS Converging Architecture with IMS Signaling analysis to achieve QoS by the intent of providing ubiquitous high-speed wireless data access, and consequently, attracting a wider user base [7].In this paper we have analyzed two different networks;one is based on 3G and other on WiMAX with same speed as well as with different speed by using Qualnet simulator 6.1 simulator. Qualnet provides scalable simulation of 3G and WiMAX network that's show performance and comparison of results through various graphs.

\section{Wimax AND 3G NETWORK BASED SYSTEM}

WiMAX is based on Wireless Metropolitan Area Network (WMAN). WiMAX system uses OFDM in the physical layer. OFDM is based on the adaptive modulation technique in non-line-of-sight (NLOS) environments. Base stations of WiMAX can provide communication without the need of line-of-sight (LOS) connection. WiMAX base station has enough available bandwidth so at a time it can serve large number of subscribers and also cover large area range. In order to provide the mobile users with the requested multimedia services and corresponding quality of service (QoS) requirements, different radio access technologies will be integrated to form a heterogeneous wireless access network.Mobile WiMAX is interworked with the already well-established 3G cellularNetworks such as Universal Mobile Telecommunications System (UMTS) or the Code Division Multiple Access based CDMA2000 system, it is more likely that WiMAX may become an essential partner of the future 4G networks [6]. The IEEE 802.16 worldwide interoperability for microwave access (WiMAX) promises to provide high data rate broadband wireless access services. The IP Multimedia Subsystem (IMS) seems to be the technology that will prevail in Next Generation Networks (NGN s), since the interworking environment and the service exibility that this technology offers to the currently deployed wireless broadband technologies makes it appealing to users, service developers and network operators [7]. The complementary coverage area and data rate characteristics of WiMAX and $3 \mathrm{G}$ networks motivate further exploration of their interworking with the intent of providing ubiquitous high-speed wireless data access, and consequently, attracting a wider user base. It is suggested that these mobile networks will be integrated by a high-bandwidth IP-based core network and a variety of heterogeneous wireless access technologies such as universal mobile telecommunications system (UMTS) or WiMAX. Mobile terminals will be able to access different multimedia applications and advanced services while roaming across zones covered by different access technologies. The wireless research community is investigating new ways to facilitate interworking between these technologies. Currently, the Third Generation Partnership Project (3GPP) is developing a feasibility study on providing seamless service continuity between UMTS and WLAN (wireless local area network) [8]. The Universal Mobile Telecommunication System (UMTS) provides high mobility with wide area coverage and supports low to medium data rates. However, UMTS data rates are not sufficient to satisfy data-intensive applications and the service charge is high. A research trend which aims to integrate UMTS and Wireless Local Area Network (WLAN) to benefit the high data rate and low cost of WLAN has much attracted research community and standardization bodies for the last few years. Recently, the Worldwide Interoperability for Microwave Access (WIMAX), a common name associated to the IEEE 802.16a/d/e wireless MAN standard [9], which provides specifications for an air interface for fixed, portable and mobile broadband wireless access networks, has not only addressed the last mile problem but also supported the nomadic and mobile clients on the go over the extended coverage area of cellular network. The 4G wireless network will integrate existing and new radio access networks that will work together to transparently offer the mobile users the need services with the corresponding QoS requirements. This will definitely bring several advantages for all parties including mobile users, network providers and service providers. On one hand, users will benefit from the coverage and characteristics provided by the different networks by allowing them to seamlessly connect, at any time and any place, to the access network that offers the "best" possible quality and the most economical service, while network and service providers will deploy the most economical technologies and infrastructures based on the offered services [10]. To compensate ubiquity and interoperability problems between these different networks, we should study procedure of handover management which insure for mobile user to move from one network to another without interrupt his active communication [11]. An attractive wireless technology for VoIP is Worldwide Interoperability for Microwave Access (WiMAX) specified by IEEE 802.16 standard aimed at providing wireless access over long distances in a variety of ways from point-to-point communication to mobile cellular access. WiMAX provides wide coverage area with lower cost of network deployment. The coverage area of a single WiMAX cell is around 30 to $50 \mathrm{Km}$ and its speed is up to $40 \mathrm{Mbps}$ [12]. The energy consumed during communication is more dominant than the energy consumed during processing. So, the communication system must be energy efficient by optimizing the energy consumption at different states of the communication. This wastes battery power, and thus the lifetime of the wireless nodes decreases. Since batteries provide limited power to operate the nodes, a general constraint of wireless communication is the short lifetime of mobile terminals [14]. As the modern high-speed data applications tend to impose a challenge on the bandwidth 
limitations of existing $3 \mathrm{G}$ cellular networks [16], a strong need for efficient mechanisms for interworking these with WLAN technologies arise.

\section{III.Simulation Setup}

We have used the Qualnet simulator 6.1 for our work. We have analyzed two differentnetworks, one is based on 3G and other on WiMAX with same speed as well as with different speed. Qualnet provides scalable simulation of $3 \mathrm{G}$ and WiMAX network that's show performance and compression of results through various graph such as Throughput, average end to end delay, average jitter and total packets received. Table I shows the parameters for new simulation design of the scenario for provides scalable simulation of $3 \mathrm{G}$ and WiMAX network at same and different speed.

TABLE I. Simulation Parameters

\begin{tabular}{|l|l|}
\hline Parameters & Values \\
\hline BS range radius (m) & 1000 \\
\hline Antenna Type & Omni directional \\
\hline Terrain-Dimensions (m) & $1500^{*} 1500$ \\
\hline Mobility Model & Random Waypoint \\
\hline Channel Bandwidth (MHZ) & 20 \\
\hline Energy Model & Generic \\
\hline Frame Duration (s) & 1 \\
\hline FFT Size & 1024 \\
\hline MS Velocity (MS $\left.{ }^{-1}\right)$ & $50,20-30$. \\
\hline BS Transmitted Power (dbm) & 20 \\
\hline SS Transmitted Power (dbm) & 20 \\
\hline Simulation Time (s) & 600 \\
\hline Traffic & CBR \\
\hline No. Of Packet Sent & 200 \\
\hline Pause Time (s) & 30 \\
\hline Radio Type & $802.16 \mathrm{e}$ \\
\hline
\end{tabular}

\section{Simulation And Analysis}

\section{a. WiMAX Scenario:}

In this paper, we have created a network architecture scenario by using Qualnet 6.1 simulator. We have designed network scenario which having central node called Hub and 25 nodes with CBR application, in which 3 nodes (node id-8, 17, 25) are base Stations. These base stations are connected through hub by wired link. CBR applications are used as a constant bit rate for data traffic which keeps the bit rate same throughout the complete process. Each base station is connected with seven mobile nodes through wireless network. There are six CBR traffic sources used in the scenario, which provides data transmission from source to destination node. All mobile stations moves with same speed as $50 \mathrm{mps}$ and varies from $20 \mathrm{mps}$ to $50 \mathrm{mps}$ by using random wave point model with pause of 30 seconds. Network architecture of this scenario with same speed is given in figure1.

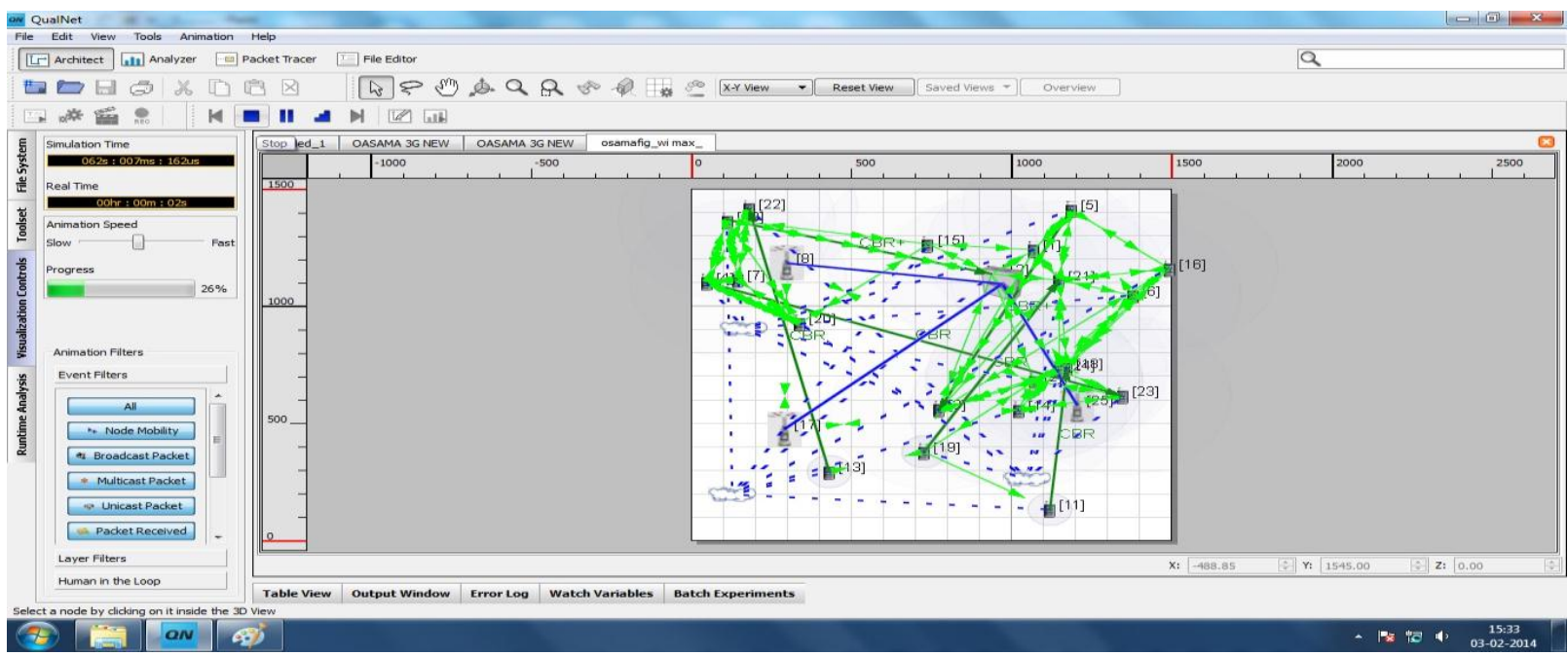


Figure 1: Scenario of WiMAX

\subsection{G Scenario}

In this scenario, we have created network architecture using UMTS devices. The architecture has one Gateway GPRS Support Node(GGSN), one Service GPRS Support Node(SGSN), one Home Location Register(HLR) and oneRadio NetworkController(RNC), threenode B's, all connected with wired link. Each Node B is connected to seven UEs through wireless network, in total 28 UEs are used. There are six CBR traffic sources are used in the scenario, which provides data transmission from source to destination node. All mobile stations moves with same speed as $50 \mathrm{mps}$ and varies from $20 \mathrm{mps}$ to $50 \mathrm{mps}$ by using random wave point model with pause of 30 seconds Some CBR applications have been used between UEs. Thenetwork architecture of this scenario is given in figure 2 .

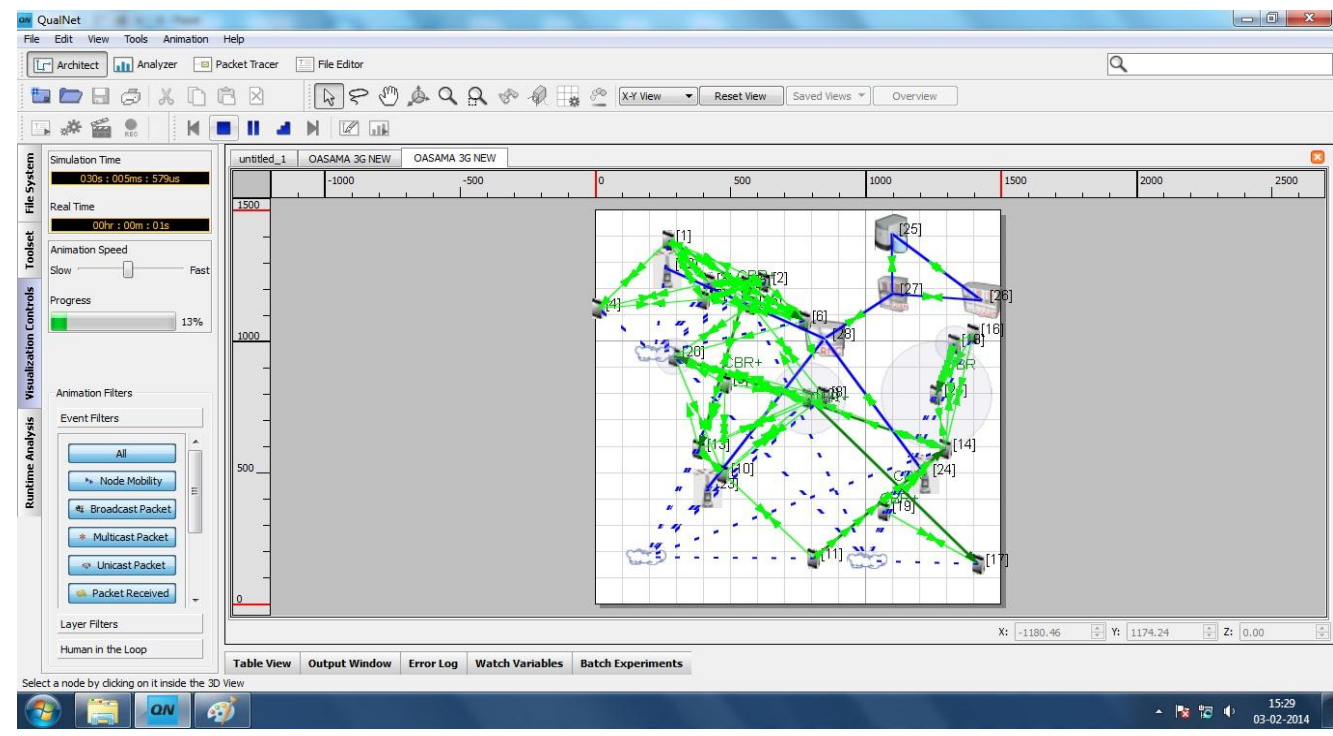

Figure 2: Scenario of $3 \mathrm{G}$

\section{Simulation Result}

The different types of scenarios are shown in this paper that covers different parameter and comparison of WiMAX and 3G. The Comparative analysis of the result that we evaluated through the simulation tool after running the scenarios are is shown in figure. 3 and figure.9.

5.1 Average Jitter:Jitter is the undesired deviation by different data packets that reached the destination and can introduce other undesired effects in audio signals and loss of transmitted data between network devices. It is the amount of variation in latency or response time, which is usually measured in milliseconds or sometime seconds for a better performance, jitter should be always low in a communication network. The graph for the average jitter is calculated under network layer.

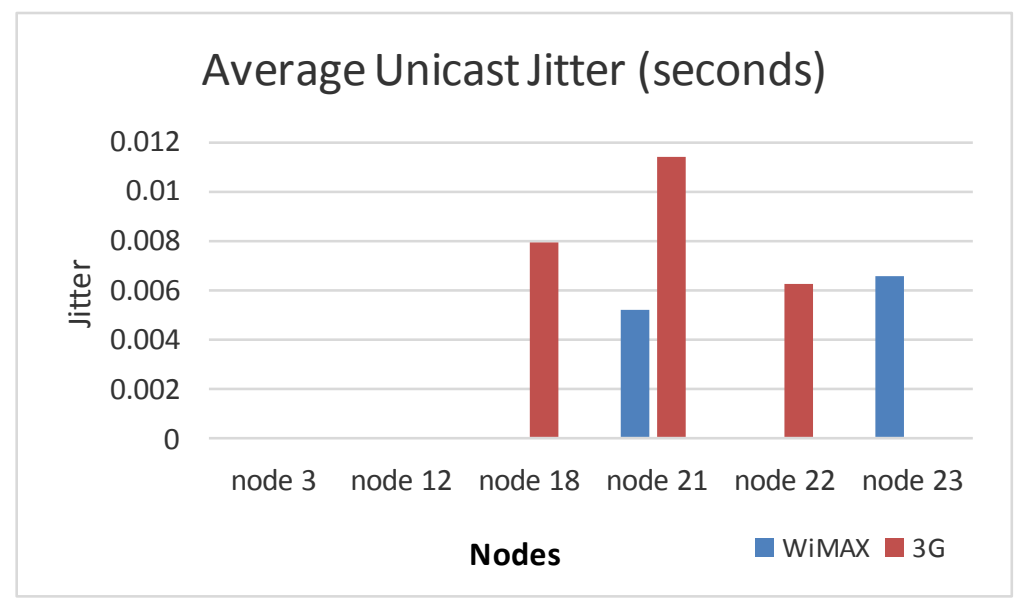


Figure 3: Graph of Average Jitter

Figure. 3 shows that jitter is very low in WiMAX as compared to $3 \mathrm{G}$ in both condition either with same speed and different speed, which shows that WiMAX performs less latency to deliver data as compared to 3G.

5.2 Unicast Received Throughput : Unicast throughput refers to the volume of data that can flow to a single destination identified by a unique address. It is usually measured in bits per second or in data packets per second. High throughput is always desirable in any communication network. The graph for the throughput is calculated by CBR Server under the application layer.

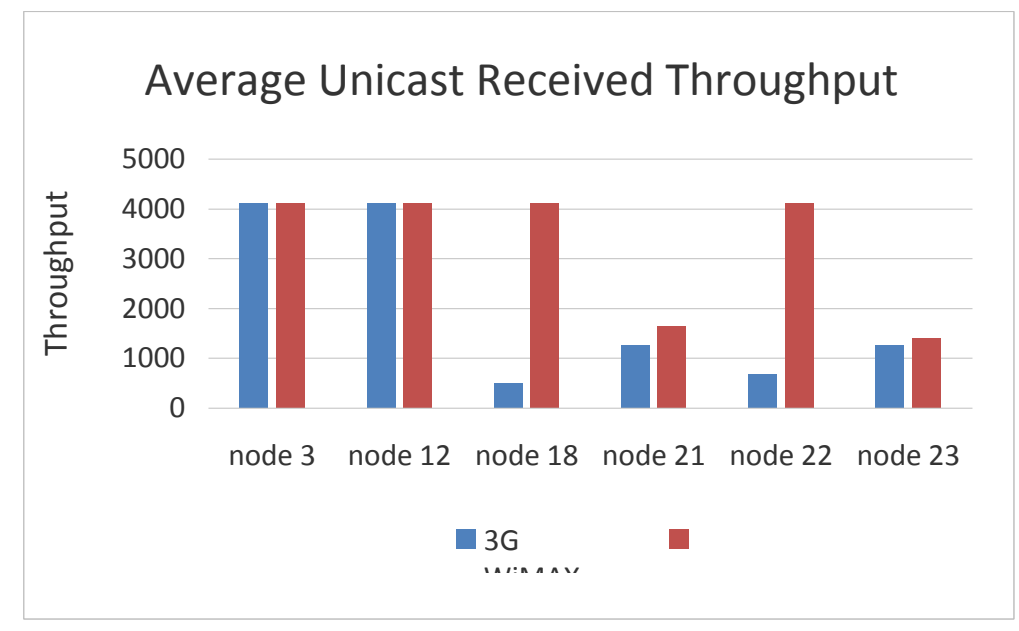

Figure 4: Graph of Unicast Received Throughput

Figure. 4 indicates that the throughput of WiMAX is greater as compared to $3 \mathrm{G}$ in both condition either with same speed and different speed.

5.3 Average Delay: The delay refers to the amount of time taken by a data bit to travel across the network from source to destination. The graph for average delay is calculated under the network layer. It is measured in seconds.

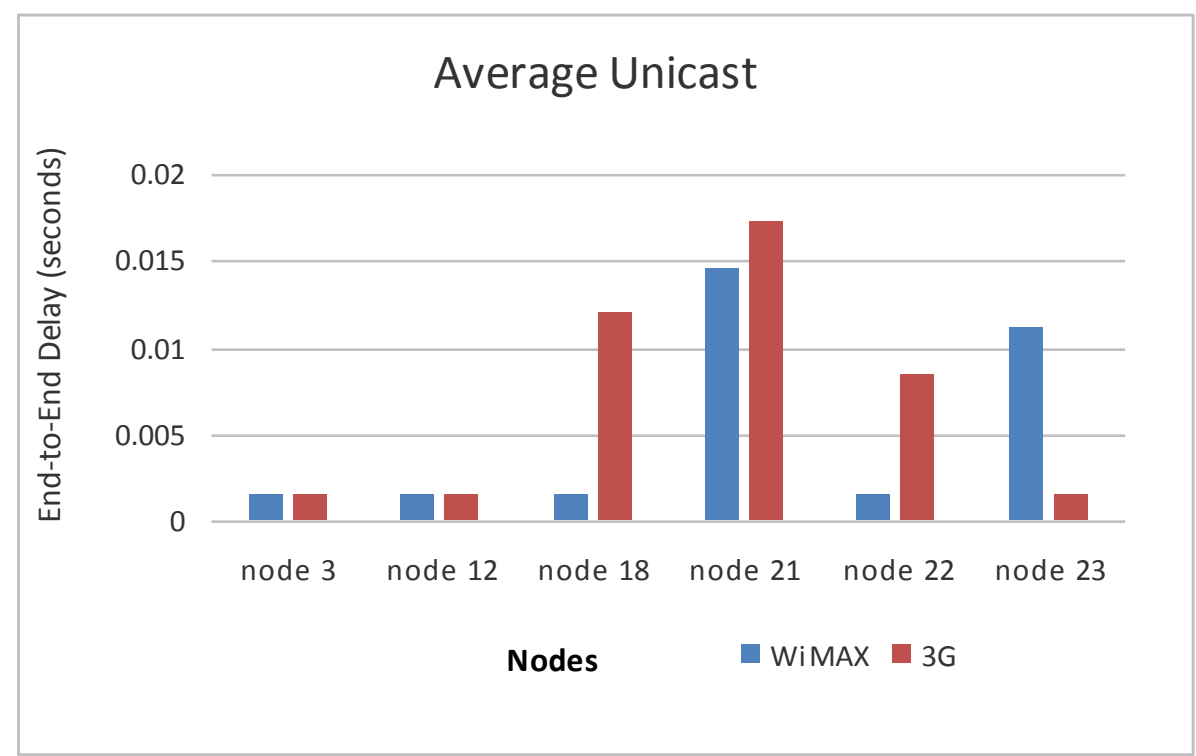

Figure 5: Graph of Average End to End Delay

The figure 5 shows that the average delay of WiMAX is very less as compared to 3G. It means WiMAX has greater speed to send the data from source to destination as compared to $3 \mathrm{G}$ in both condition either with same speed and different speed. 
5.4 Average Queue Length - Queue length is the total no of threads per second that are waiting in queue in order to access the channel since the application started. The graph for average queue length is calculated under the network layer. It is measured in Bytes and scheduled in form of FIFO order.

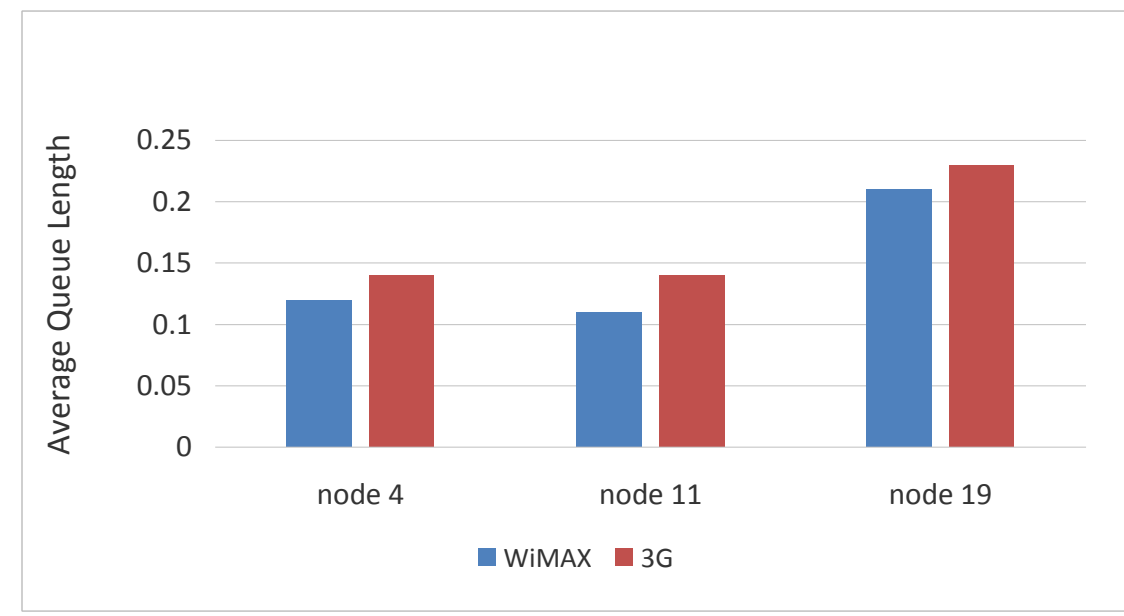

Figure 6: Graph of Average Queue Length

It is observed in figure.6 that the average queue length of WiMAX is less than $3 \mathrm{G}$. Here, it is clear that, in WiMAX, packets access the channel with faster speed, so they don't have to waste more time in waiting to access the channel.

5.5 Total Unicast Data Sent and Received-Total unicast data sent refers to the total number of data sent from source to destination and total unicast data received refers to the total number of data received from the same. The graph for total unicast data sent and received is calculated by CBR client and CBR server under application layer. It is measured in bytes. Figure. 7 shows that the total number of packets received in WiMAX are more as compared to $3 \mathrm{G}$.

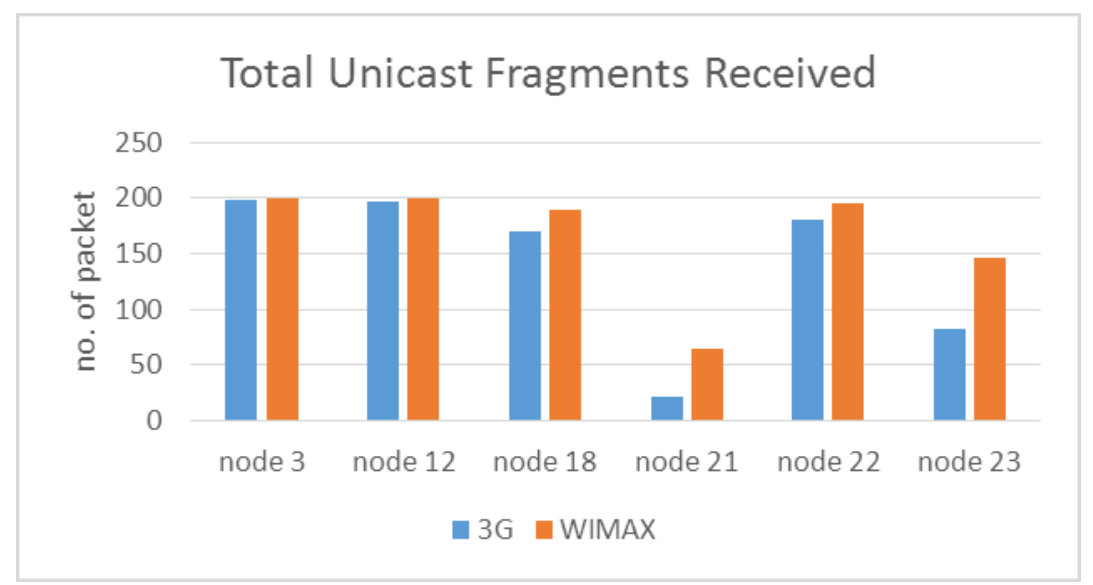

Figure 7: Graph of Total Unicast Data Received 
. 5.6 Signal Received with Error- It refers to the number of incoming signals that failed to receive by the destination. Graphs of WiMAX and 3G for this metric are calculated in sequence 802.16 and UMTS under the physical layer.

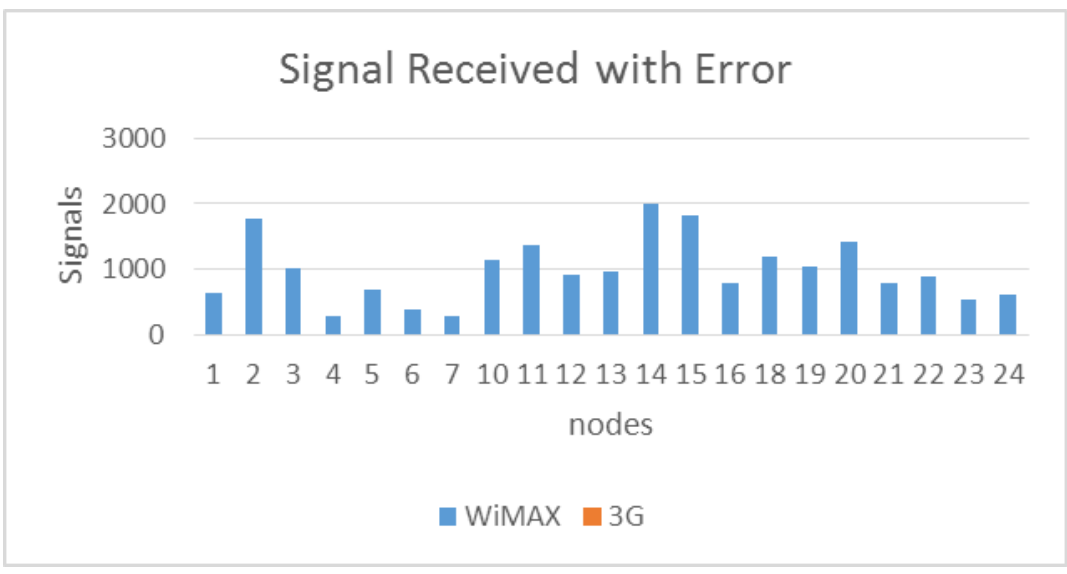

Figure 8: Graph of Signal Received with Error

From the figure.8, we observed that, in WiMAX, at physical layer the value of error signals are 958.87 units, but in $3 \mathrm{G}$, there is no signal with error received at physical layer.

\section{Conclusion}

On comparing the result it is concluded that WiMAX is more efficient than $3 \mathrm{G}$ in both the cases either in same speed or in different speed for the users and service provider. WiMAX network has high performance which has observed by different parameters such as high throughput, less energy consumption, small queue length, low jitter and less delay. WiMAX has better performance than $3 \mathrm{G}$ with amount of data were sent to both the networks by calculating the server parameters. WiMAX received same numbers of data as was sent while the no of data received by $3 \mathrm{G}$ is less than the total no of data sent It is also observed that the performance of WiMAX network increased than $3 \mathrm{G}$ network by varying the speed of subscriber station. Further work may be focused on mobility of subscriber stations to the same network by using flags for proving path to the mobile stations that will cover more area and provide better communication.

\section{References}

[1] Anil Goyal and Dharmendra Kumar Jhariya; "Simulation and performance Analysis of Ad-hoc Routing Protocol in Wi-Fi \&WiMAX networks using QualNet", International Journal of Electronics and Computer Science Engineering, ISSN- 2277-1956, Vol-1, No-3, pp. 1593-1601, July 2012.

[2] Intel;"'UnderstandingWiMAX and 3 G for portable/Mobile Broadband Wireless", Technical White Paper, dec 2004.

[3] "Comparing mobile WiMAX, 3G and Beyond", White Paper, WiMAX forum, 2007.

[4] Fasasi .A. Adebari and Olalekan Bello. "Mobile WiMAX as a Next Generation Broadband Wireless Networks" International Journal of Science and Technologyvol 2, no. 1, pp. 77-80, Jan 2013.

[5] Sarra Ben Oubira, MounirFrikha and Sami Tabbane, "Loose coupling Approach for UMTS/WiMAX Integration" in Proceedings of the IFIP Wireless Days Conference 2011, Niagara Falls, ON, Canada, 10-12 October 2011, pp. 13.

[6] Kumudu S. Munasinghe, and Abbas Jamalipour, "Interworked WiMAX-3G Cellular Data Networks:An Architecture for Mobility Management and Performance Evaluation", IEEE Transactions on Wireless Communications, Vol. 8, Issue: 4, pp. 1847-1853, April 2009.

[7] Vijayalakshmy. G and Sivaradje. G, "WiMAX-UMTS Converging Architecture with IMS Signalling analysis to achieve QoS" International Journal of Innovative Research in Science, Engineering and Technology, Vol., Issue 4, pp. 1188-1194, April 2013

[8] FangminXu, Luyong Zhang and Zheng Zhou, "Interworking of Wimax and 3GPP networks based on IMS [IP Multimedia Systems (IMS) Infrastructure and Services]", Communications Magazine, IEEE Volume:45, Issue: 3 , pp. 144-150, March 2007.

[9] Quoc-Thinh Nguyen-Vuong, Fiat, L. and Agoulmine, N., "An Architecture for UMTS-WIMAX Interworking", in the 1st International Workshop on Broadband Convergence Networks, Vancouver,.BcN 2006, pp. 1-10,7 April 2006.

[10] Charles Sarraf, FirasOusta, NidalKamel and MohdZuki, "Mapping of QoS between UMTS and WiMAX in Tight Coupling Heterogeneous Wireless Network", International Journal of Soft Computing and Software Engineering, vol-2, no.-3, pp. 1-14, March 2012. 
[11] Haji, A. , Ben Letaifa, A. and Tabbane, S., "Integration of WLAN, UMTS and WiMAX in 4G", in 16th IEEE International Conference on Electronics, Circuits, and Systems, YasmineHammamet,. ICECS 2009, pp. 307-310,13-16 Dec 2009.

[12] Jadhav, S. ,Haibo Zhang and Zhiyi Huang, "Performance Evaluation of Quality of VoIP in WiMAX and UMTS", in 12th International Conference on Parallel and Distributed Computing, Applications and Technologies (PDCAT), 20-22 Oct 2011, pp. 375-380.

[13] Perkins, C., Belding-Royer, E. and Das, S.; "Ad hoc on-Demand Distance Vector (AODV) Routing”, RFC 3561 (rfc3561) - Page 137, July2003.

[14] DharamVir, Dr. S K Agawal, Dr. S A Imam; “A Simulation Study on Node Energy Constraints of Routing Protocols of Mobile Adhoc Network Use of QualNet Simulator", International Journal of Advanced Research in Electrical, Electronics and Instrumentation Engineering, Vol-1, Issue: 5, pp. 401-410, Nov 2012.

[15] IEEE, "Air Interface for Fixed and Mobile Broadband Wireless Access Systems," IEEE P802.16e/D12, Feb. 2005.

[16] A. K. Salkintzis, C. Fors, and R. Pazhyannur,"WLAN-GPRS integration for next-generation mobile data networks," in IEEE Wireless Commun., vol. 9, pp. 112-124, 2002.

[17] K. Munasinghe and A. Jamalipour, "A 3GPP-IMS based approach for converging next generation mobile data networks," in Proc. IEEE Int. Conf. on Commun. (ICC2007), Glasgow, June 2007.

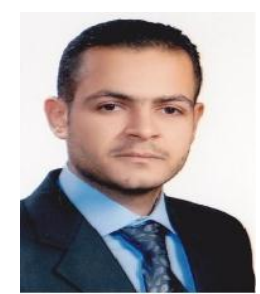

\section{OsamahThamar AL-Zubaidi}

He Received his bachelor of Electronic and Communication Engineering Technical Najaf Collage From Iraq in2011.He is currently pursueinghis masters in engineering from department of Electronics and Communication Engineering,SHIATS.

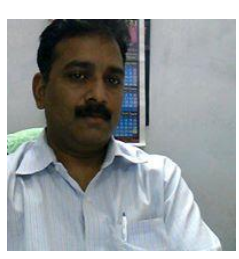

\section{Dr. Rajeev Paulus}

Working as an Assistant Professor in the Department of Electronics and Communication Engineering in SHIATS, Allahabad.He received the degree of M.Tech from MNNIT, Allahabad. He received the degree of Ph.D. from SHIATS, ALLAHABAD. He has presented and published various research papers in national and international Journals andconferences. He is currentlyfocusing on thearea of wireless sensor and adhoc network, high speed data network.

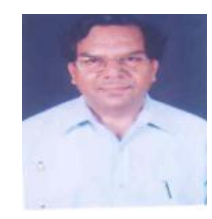

\section{Prof.A.K. Jaiswal}

Working as Prof. and Head of ECE Dept. SHIATS-Allahabad. He Obtained M.Sc. (Tech) in Electronic \& Radio Engg. From J. K. Institute of Technology Allahabad University in1967. He guided various projects \& research at undergraduate \& postgraduate level. He has more than 35years Industrial, research \& Teaching experience and actively involved in research and publications. His area of interest includes Optical Networks and satellite communication. His achievement includes receipt of bronze shield (national award) for developing miniature indicator/controller for process industries. He also worked as investigator for developing and promoting optical fiber based systems in a project sponsored by DOE, govt. of India and UNDP.

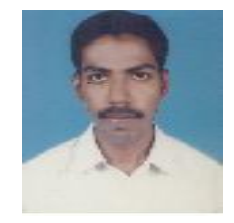

\section{Er.A. Ashok}

He received his B.Edegree in the year 2008 from Anna University, Chennai and A.Ashok is working as Assistant professor in the Department of Electronics and Communication Engineering completed hisM.Tech "Communication System Engineering" from SHIATS, Allahabad. His research is focus on Digital Signal Processing \& its Applications.

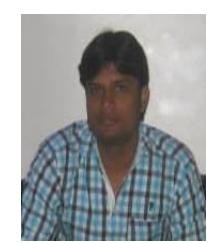

\section{Ashishshukla}

He Received his bachelor of Electronic and Communication Engineering from Dr.M.C Saxena college of engineering. He is currently pursueing his masters in engineering from department of Electronics And Communication Engineering, SHIATS 